\title{
A difference boosting neural network for automated star-galaxy classification
}

\author{
N. S. Philip ${ }^{1}$, Y. Wadadekar ${ }^{2,3}$, A. Kembhavi ${ }^{3}$, and K. B. Joseph ${ }^{1}$ \\ 1 Cochin University of Science and Technology, Kochi - 682 022, India \\ 2 Institut d'Astrophysique de Paris, 98bis Boulevard Arago, 75014 Paris, France \\ 3 Inter University Center for Astronomy and Astrophysics, Post Bag 4, Ganeshkhind, Pune - 411 007, India
}

Received 3 May 2001 / Accepted 5 February 2002

\begin{abstract}
In this paper we describe the use of a new artificial neural network, called the difference boosting neural network (DBNN), for automated classification problems in astronomical data analysis. We illustrate the capabilities of the network by applying it to star galaxy classification using recently released, deep imaging data. We have compared our results with classification made by the widely used Source Extractor (SExtractor) package. We show that while the performance of the DBNN in star-galaxy classification is comparable to that of SExtractor, it has the advantage of significantly higher speed and flexibility during training as well as classification.
\end{abstract}

Key words. galaxies: fundamental parameters - stars: fundamental parameters - methods: statistical methods: data analysis

\section{Introduction}

Automated classification using neural networks has recently found several applications in astronomy. These range from classification of stellar (e.g. Singh et al. 1998) and galactic (Folkes et al. 1996) spectra, differentiating between stars and galaxies from imaging data (e. g. Odewahn et al. 1992), to detecting defects on CCDs (Rogers \& Riess 1994).

Two powerful neural network paradigms (and their variants) have been popular in the astronomical community for addressing classification problems. These are the Back-propagation algorithm (see McClelland \& Rumelhart 1982) and the Self-Organizing Map (SOM; Kohonen 1982). The basic difference between the two is that SOM can organize itself into clusters unsupervised, meaning that no prior definition of the correct output for an input vector is needed. On the other hand, backpropagation requires a carefully selected training set for successful convergence. Both approaches have their own advantages and disadvantages depending upon the application. When an input is presented, SOM tries to associate it with a model vector that has features that best match with those of the input vector. If no such model vector is found, SOM assigns a new label to the object and considers it as the model vector of a new class of objects. SOM is therefore good in situations where the user does not know

Send offprint requests to: Y. Wadadekar,

e-mail: wadadeka@iap.fr the possible classes of objects a priori. However, SOM has the disadvantage that it often classifies objects into different groups when in fact they belong to a single group. In the astronomical context, this often happens due to time-dependent variations in features associated with the data, e. g. variations in seeing conditions. Nevertheless, a number of variants of SOM have appeared with different adaptation rules, distance measures and structures of the map interconnections (Kohonen 1995).

Back-propagation, on the other hand, is most suitable in situations where one knows a priori the possible classes that an object can belong to, and wants to classify objects with a minimum error in the classification. From a computational perspective, back-propagation is a kind of function fitting process in which the parameters, generally known as the connection weights, are modified so that the network is able to map the system in terms of a nested sum of products of its node functions, which are generally sigmoid functions. The back-propagation network requires a training set, a test set and often a validation data set. In the training process the connection weights are updated in proportion to the negative gradient of the error surface. The network passes through the same set of data several times, lowering the overall error in each pass. Since in many astronomical classification problems the human observer is able to classify sources into their possible classes directly, and only requires the machine to behave as a faster classifier, back-propagation and its variants have been widely used for classification of astronomical images 
and spectra. Another advantage of the back-propagation network over SOM is that the user has full control over the relevant parameters to be used for the classification, and can therefore prevent the system from being misled by irrelevant features in the training data. Raw data is preprocessed to extract the significant parameters which then become the input for the neural network.

Automated and accurate classification of objects into stars and galaxies from optical (and near infrared) imaging data is an issue of considerable interest. Artificial neural network based approaches to the star galaxy classification problem include SOM (Miller \& Coe 1996), decision tree induction (Weir et al. 1995) and back propagation, which is the basis for SExtractor, a widely used tool for stargalaxy separation (Bertin \& Arnouts 1996). One of the drawbacks of classification tools such as SExtractor that employ back propagation is that it is difficult to modify them for specific needs. With the advent of a number of visible and near-infrared sky surveys, with varying sensitivities and observing conditions, it is often desirable for the user to be able to specify the optimum parameters for classification after experimenting with several possibilities. Trying new parameters on a general purpose tool like SExtractor is computationally very time consuming. The objective of this paper is to present a tool that can do the training at a faster rate, so that the network can be rapidly tested with different sets of classification parameters to discover the optimum parameter set. The short training time makes the network very flexible and its potential astronomical applications are much wider than the star-galaxy classification problem discussed here.

\section{The Difference Boosting Neural Network (DBNN)}

DBNN is based on a powerful and intuitive procedure originally developed and used by mathematicians (Laplace 1812) for sensible classification of objects. Named after its inventor Bayes (1763), Bayes' theorem, according to Laplace, is the mathematical expression of common sense. Bayes' theorem computes the conditional probability for the occurrence of an event, given that another event which could lead to this event has occurred (for an introduction to Bayes' theorem see Loredo 1990). In complex problems, computation of the Bayesian probability becomes a laborious process. However, a variant of the Bayesian classifier known as the naive Bayesian classifier (Elkan 1997) is able to compute the Bayesian probability with reasonable accuracy under the assumption that, given the class, the attribute vectors (arrays of parameter values) are independent. However, in practice this independence assumption is frequently invalid and the performance of the network degrades when there are correlated attribute vectors.

The Difference Boosting Algorithm (DBNN) (Philip \& Joseph 2001) is a computationally less intensive Bayesian classifier algorithm than its peers, and is closely related to the naive Bayesian classifier. DBNN, however, does not strictly follow the independence of attributes as a basic
Table 1. Truth table of the XOR gate.

\begin{tabular}{lll}
\hline \hline Input 1 & Input 2 & Output \\
\hline 0 & 0 & 0 \\
\hline 0 & 1 & 1 \\
\hline 1 & 0 & 1 \\
\hline 1 & 1 & 0 \\
\hline
\end{tabular}

criterion and allows some correlation between the attributes. It does this by associating a threshold window with each of the attribute values of the sample. The threshold window demands that all the attribute values be in the range specified by the training set for each class of the sample. When any of the attribute values is outside the range specified by the threshold function, the confidence in the classification is penalized by a certain factor.

A popular example manifesting the correlation between input parameters is the XOR gate. A typical XOR gate has two digital inputs and one digital output. A digital state has only two possible values represented as high or low state. The XOR gate behaves such that its output is a high only when the two inputs are dissimilar, as shown in Table 1. Although the conditions appear to be simple, this is a case where the conditional independence is violated. For example, from the knowledge of the value of only one of the inputs, it is not possible to have any preferred knowledge about the class of the object. The actual class can be assigned only when both the inputs are known together. Since only the value of one of the inputs is used by the naive Bayesian classifier at a time, it is not able to produce a confidence level better than $50 \%$ (both alternatives equally likely) on such data. However, since DBNN takes into account the values of the other parameters by the use of the window function, it is able to give an accurate representation of the output states. We then say that the network has learned the XOR problem.

Some other advantages of the DBNN algorithm are its explicit dependence on probability estimates, its ability to give an estimate of the confidence value of a prediction and greater training speed. For the particular application to star galaxy classification, the DBNN gives good results with fewer input parameters than SExtractor. Philip \& Joseph (2001), compared results from DBNN with the results obtained by Schiffmann et al. (1994) on sixteen other network models. While Schiffmann et al. (1994) report an average training time of $\sim 12$ hours on the dataset they used, DBNN on the same dataset took only about $10 \mathrm{~min}$ for training. While their best result from the eighteen models produced an accuracy of $98.48 \%$ on independent test data, DBNN gave an accuracy of $98.60 \%$.

One of the motivations for the DBNN classifier is that the human brain looks for differences rather than details when it is faced with situations that require distinction between almost identical objects. While the standard Bayesian method is very elaborate and takes every possibility into consideration, and the naive Bayesian ignores 
all possible correlations between attribute values, DBNN is an attempt to have the best of both worlds by highlighting only the differences.

\section{Working of the DBNN}

The working of the DBNN may be divided into three units. The first unit computes Bayes' probability and the threshold function for each of the training examples. The second unit consists of a gradient descent boosting algorithm that enhances the differences in each of the examples in an attempt to minimize the number of incorrectly classified cases. At this stage, boosting is applied to the connection weights for each of the probability components $P\left(U_{m} \mid C_{k}\right)$ of the attribute $U_{m}$ belonging to an example from the class $C_{k}$. Initially all the connection weights are set to unity. For the correctly classified object, the total probability $P\left(U \mid C_{k}\right)$, computed as the product of component probabilities will be a maximum for $C_{k}$, the class of the object given in the training set. For the wrongly classified examples, for each of the component probability values, the associated weights are incremented by a factor $\delta W_{m}$ which is proportional to the difference in the total probability of membership of the example in the stated class and that in the wrongly classified class. The exact value is computed as

$\delta W_{m}=\alpha\left(1-\frac{P_{k}}{P_{k}^{*}}\right)$

in a sequence of iterations through the training set. Here $P_{k}$ is the computed total probability for the actual class of the object and $P_{k}^{*}$ that of the wrongly classified class. The parameter $\alpha$ is a learning constant functionally similar to the learning constant in the back-propagation algorithm. It thus defines the rate at which the algorithm updates its weight parameter. The third unit computes the discriminant function (Bishop 1999) $P\left(C_{k} \mid U\right)$ as:

$P\left(C_{k} \mid U\right)=\frac{\prod_{m} \hat{P}\left(U_{m} \cap C_{k}\right) W_{m}}{\sum_{j} \prod_{m} \hat{P}\left(U_{m} \cap C_{j}\right) W_{m}}$.

Here $\hat{P}\left(U_{m} \cap C_{k}\right)$ stands for $P\left(U_{m} \cap C_{k}\right) / P\left(C_{k}\right)$ which from the axioms of set theory is equivalent to $P\left(U_{m} \mid C_{k}\right)$.

In the implementation of the network, the actual classification is done by selecting the class corresponding to a maximum value for the discriminant function. Since this value is directly related to the probability function, its value is also an estimate of the confidence with which the network is able to do the classification. A low value indicates that the classification is not reliable. Although a network based on back-propagation also gives some probability estimates on the confidence it has on a classification scheme, these are not explicitly dependent on the probabilities of the distribution. Thus while such networks are vulnerable to divergent training vectors that are invariably present in training samples, DBNN is able to assign low probability estimates to such vectors. This is especially significant in astronomical data analysis where one has to deal with variations in the data due to atmospheric conditions and instrumental limitations. Another significance of the approach is the simplicity in the computation. DBNN can be retrained with ease to adapt to the variations in the observations enabling one to generate more accurate catalogs.

In the following section, we describe the use of the DBNN technique to differentiate between stars and galaxies in broadband imaging data. We chose to illustrate the capabilities of the DBNN by addressing the star-galaxy classification problem for the following reasons:

1. A widely used benchmark implementation of the back propagation algorithm is already available for tackling this problem in SExtractor.

2. High quality imaging data has recently become available from ongoing optical surveys. The number of sources detected by such surveys is large enough for us to construct moderately large training and test sets from uniformly high quality data. The data we use here is publicly accessible and our results can therefore be verified and extended by other researchers.

3. Construction of a reasonably accurate training set is possible from visual examination, given our experience with optical imaging data.

\section{Separating stars and galaxies}

The star-galaxy classification problem addresses the task of labeling objects in an image either as stars or as galaxies based on some parameters extracted from them. Classification of astronomical objects at the limits of a survey is a rather difficult task, and traditionally has been carried out by human experts with intuitive skills and great experience. This approach is no longer feasible, because of the staggering quantities of data being produced by large surveys, and the need to bring objectivity into the classification, so that results from different groups can be compared. It is thus necessary to have machines that can perform the task with the efficiency of a human expert (but at much greater speed) and with robustness in the classification, over variations in observing conditions.

Processing the vast quantities of data produced by new and ongoing surveys and generating accurate catalogs of the objects detected in these surveys is a formidable task, and reliable and fast classifiers are much in demand. Following the work by Odewahn et al. (1992), there has been a growing interest in this area in the past decade. SExtractor (Bertin \& Arnouts 1996) is a popular, publicly available general purpose tool for this application. SExtractor accepts a FITS image of a region of the sky as input and provides a catalog of the detected objects as output. It has a built-in back propagation neural network which was trained once for all by the authors of SExtractor using about $10^{6}$ simulated images of stars and galaxies, generated under different conditions of pixel-scale, seeing and detection limits. In SExtractor an object is classified quantitatively by a stellarity index ranging from zero to 
unity, with index zero representing a galaxy and unity representing a star. The stellarity index is also a crude measure of the confidence that SExtractor has in the classification. A stellarity index of 0.0 or 1.0 indicates that SExtractor confidently classifies these objects as galaxy and star respectively while a stellarity index of 0.5 indicates that SExtractor is unable to classify the object. The input to the neural network used by SExtractor consists of nine parameters for each object, extracted from the image after processing it through a series of thresholding, deblending and photometric routines. Of the nine input parameters, the first eight are isophotal areas and the ninth one is the peak intensity for each object. In addition to these nine parameters, a control parameter, the seeing full width at half maximum ( $F W H M)$ of the image, is used to standardize the image parameters against the intrinsic fuzziness of the image due to the seeing conditions. In practice, some fine tuning of this control parameter is required for obtaining realistic output from the network, due to the wide range of observing conditions encountered in the data. A scheme for carrying out such tuning is described in the SExtractor manual.

Among other packages proposed recently for stargalaxy classification in wide field images is NExtractor (NExt) by Andreon et al. (2000). NExt claims to be the first of its kind that uses a neural network both for extracting the principal components in the feature space, as well as for classification. The performance of the network was evaluated over twenty five parameters that were expected to be characteristic to the class label of the objects, and it was found that six of these parameters, namely, the harmonic and Kron radius, two gradients of the PSF, the second total moment and a ratio that involves the measures of intensity and area of the observed object were sufficient to produce optimum classification. A comparison of NExt performance with that of SExtractor by Andreon et al. (2000) showed that NExt has a classification accuracy that is as good as or better than SExtractor. The NExt code is not publicly available at the present time (Andreon, personal communication) and a comparison with DBNN is not possible.

\subsection{Constructing the training set}

The first requirement for the construction of any good classifier is a complete training set. Completeness here means that the training set consists of examples with all possible variations in the target space and that the feature vectors derived from them are distinct in the feature space of their class labels. In the context of star-galaxy classification, this means that the training set should contain examples of the various morphologies and flux levels, of both stars and galaxies, spanning the entire range of parameters of the objects that are to be later classified.

We decided to construct our training set from an $R$ band image from the publicly available NOAO Deep Wide Field Survey (NDWFS). This survey will eventu-
Table 2. Summary of the NDWFS field used for constructing training and test sets.

\begin{tabular}{ll}
\hline \hline Field Name & NDWFSJ1426p3456 \\
Filter & $R$ \\
R.A. at field center (J2000) & $14: 26: 01.41$ \\
Dec. at field center (J2000) & $+34: 56: 31.67$ \\
Field size & $36.960^{\prime} \times 38.367^{\prime}$ \\
Total Exposure time (hours) & 1.2 \\
Seeing $F W H M(\operatorname{arcsec})$ & 1.16 \\
\hline
\end{tabular}

Table 3. Values of important SExtractor parameters used in construction of the training and test sets.

\begin{tabular}{ll}
\hline \hline Parameter & Value \\
\hline DETECT_MINAREA & 64 \\
DETECT_THRESH & 3 \\
ANALYSIS_THRESH & 1.0 \\
FILTER & $N$ \\
DEBLEND_NTHRESH & 32 \\
DEBLEND_MINCONT & 0.01 \\
CLEAN & $\mathrm{N}$ \\
SATUR_LEVEL & 49999.0 \\
MAG_ZEROPOINT & 30.698 \\
GAIN & 46.2 \\
PIXEL_SCALE & 0.258 \\
SEEING_FWHM & 1.161 \\
BACKPHOTO_TYPE & LOCAL \\
THRESH_TYPE & RELATIVE \\
\hline
\end{tabular}

ally cover 18 square degrees of sky. The first data from the survey obtained using the MOSAIC-I CCD camera on the KPNO $4 \mathrm{~m}$ Mayall telescope were released in January 2001. We chose to use data from this survey because of its high dynamic range, large area coverage and high sensitivity that allowed us to maintain uniformity between the moderately large training set and numerous test sets. The training set was carefully constructed from a subimage of the $R$ band image NDWFSJ1426p3456 which has the best seeing conditions among the data currently released. Details of the image are listed in Table 2.

We used SExtractor as a preprocessor for selection of objects for the training set and for obtaining photometric parameters for classification. The values of some critical configuration parameters supplied to SExtractor for construction of the object catalog are listed in Table 3. Saturated stars were excluded from the training set by setting the SATUR_LEVEL parameter. SEEING_FWHM was measured from the point spread function (PSF) of the brightest unsaturated stars in the image. The DETECT_MINAREA parameter was set so that every selected object had a diameter of at least 1.8 times the FWHM of the PSF. DETECT_THRESH was set conservatively to 3 times 
Table 4. Details of the regions used in constructing the training and test data sets. Stars and galaxies from NDWF10 were used in training the network, while the other two data sets were used to test the performance of the network after training. Each region was $2001 \times 2001$ pixels in size.

\begin{tabular}{llllll}
\hline \hline Data Label RA (J2000) & Dec (J2000) & Stars & Galaxies & Total \\
\hline NDWF10 & $14: 26: 28.76$ & $34: 59: 19.94$ & 83 & 319 & 402 \\
NDWF5 & $14: 27: 11.23$ & $34: 50: 50.92$ & 65 & 239 & 304 \\
NDWF14 & $14: 26: 28.18$ & $35: 07: 55.69$ & 89 & 319 & 408 \\
\hline
\end{tabular}

the standard deviation of the background which was estimated locally for each source. ANALYSIS_THRESH was set to a lower value to allow for more reliable estimation of the classification parameters we used. No cleaning or filtering of extracted sources was done. DEBLEND_NTHRESH and DEBLEND_MINCONT were set by trial and error using the guidelines in the SExtractor documentation. The following parameters were obtained from descriptions of the NDWFS data products in the NOAO archives - PIXEL_SCALE, MAG_ZEROPOINT and GAIN. SExtractor computes several internal error flags for each object and reports these as the catalog parameter FLAGS. Objects with a FLAGS parameter $\geq 4$ were deleted from the training set. This ensured that saturated objects, objects close to the image boundary, objects with incomplete aperture or isophotal data and objects where a memory overflow occurred during deblending or extraction were not used.

The training set was constructed from objects satisfying the above criteria from a $2001 \times 2001$ pixel region of the image described in Table 2 . The image region we used was selected at random. The objects were largely in the Kron-Cousins magnitude range 20-26. Objects brighter than this limit are mostly saturated stars which were not used. Each object in the training set was visually classified as a star or galaxy by two of the authors working separately, after examining the radial intensity profile, surface map and intensity contours. Less than $2 \%$ of the sources were differently classified by the two authors. These discrepancies were resolved by a combined examination by both authors. It was not possible to visually classify 35 of the objects, and these were deleted from the training set. All the deleted objects are fainter than magnitude 26. Some details about the training set, named NDWF10, are given in Table 4. Visual classification of many of the brighter stars was aided by the perceptibly non-circular PSF of the image. After visual classification was complete, SExtractor classification for all sources in the training set was obtained. An object-by-object comparision of the visual and SExtractor classification showed that the latter was successful in $97.76 \%$ of the cases in reproducing the results of the visual classification (see Table 4). The number of stars in the training set is considerably smaller than the number of galaxies because of the high galactic latitude of the field and the faint magnitudes of objects in the training set.

\subsection{Obtaining optimum parameters for classification}

Once a training set is available, the next task is to select the parameters that the network will use for classification. We tested all available parameters extracted by SExtractor for their suitability as classification parameters. We also derived some new parameters from the basic parameters obtained from SExtractor. For the classification we sought parameters which were (a) not strongly dependent on the properties of the instrument/telescope and on observing conditions; (b) would not depend on photometric calibration of the data, which is not always available; and (c) resulted in the clearest separation between stars and galaxies. To meet the last requirement, we plotted each parameter against the FWHM of the intensity profile and identified the parameters which provided the best separation. After extensive experimentation with our training set data, we found that three parameters were most suitable. These are:

1. Elongation measure: this is the logarithm of the ratio of second order moments along the major and minor axis of the lowest isophote of the object. For a star, the ratio should be near unity. For our training set, this ratio is different from unity because of the slightly elliptical PSF.

2. The standardized $F W H M$ measure: this is the logarithm of the ratio of the FWHM of the object (obtained from a Gaussian fit to the intensity profile) to the $F W H M$ of the point spread function for the image.

3. The gradient parameter (slope): this is the logarithm of the ratio of the central peak count to the FWHM of the object, normalized to the FWHM of the point spread function for the image.

We trained the DBNN using the values for these parameters for the visually classified set of stars and galaxies as the training set. In Fig. 1 we show plots of the three final DBNN parameters against each other, with stars and galaxies marked differently. It is clear that excellent separation between stars and galaxies is obtained.

\subsection{Testing the network performance}

We tested the network on 2 sub-regions $(2001 \times 2001$ pixels each) of the NDWFSJ1426p3456 field. The central coordinates of the two test set images are listed in Table 4. Using a different region of the same field for testing ensures that erroneous classification due to variations in data quality is not an issue. As in the case of the training set, these sub-regions were also selected at random. The object catalogs for the test sets were constructed using the same SExtractor configuration as for the training set. DBNN marked some objects as boundary examples, meaning that their confidence level was not more than $10 \%$ above the plain guess estimate $(50 \%)$ regarding the class of the object.

In test set 1 (NDWF5), 32 out of 336 objects were deleted as they could not be classified visually. Of the 

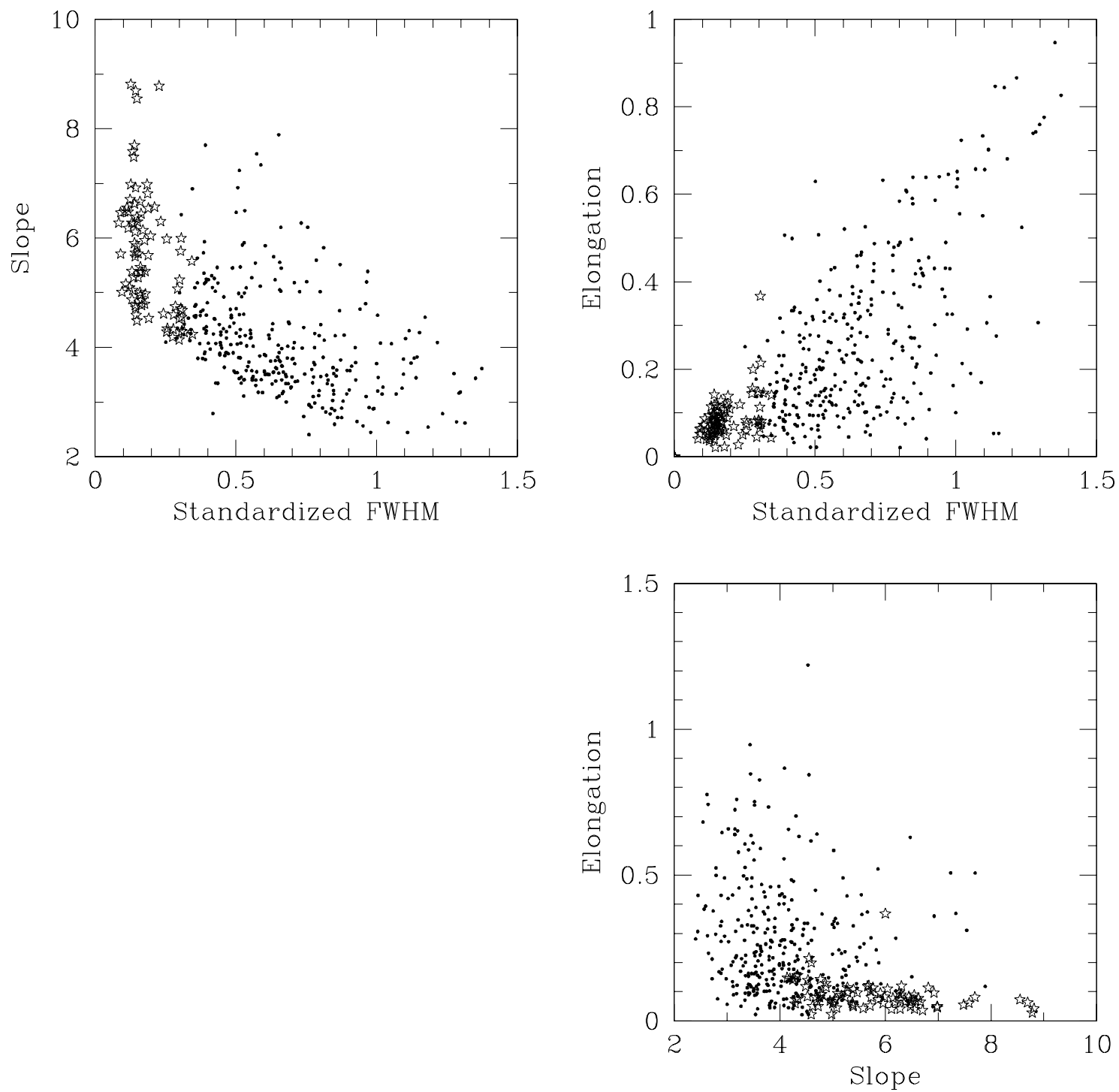

Fig. 1. The figures show clusters formed by stars and galaxies in the feature space. Galaxies are shown as dots and stars as stars.

remaining 314 objects, DBNN found 15 as marginal but classified 10 of these correctly. Two objects were misclassified. In test set 2 (NDWF14), 14 out of 422 objects were deleted for which visual classification was not possible. Of the remaining, DBNN marked 17 objects as marginal but classified 12 of these correctly. One object was misclassified. The results for the two test sets are summarized in Table 5. The classification accuracy is marginally better than that of SExtractor. The marginal superiority of DBNN, in the test set data, is not significant if some allowance is made for subjectivity in the construction of the test set. However, the fact that DBNN can obtain high classification accuracy with only 3 parameters as compared to $10(9+1$ control $)$ parameters used by SExtractor is of some importance.

\subsection{Effects of image degradation}

An important consideration is to check the performance of DBNN (and SExtractor) on low signal to noise images.
Table 5. Comparison of classification accuracy of the DBNN and SExtractor on the NDWFS data. There is no entry under DBNN for NDWF10, since this data set was used in training the DBNN network.

\begin{tabular}{lll}
\hline \hline Data Label & $\begin{array}{l}\text { Classification Accuracy } \\
\text { SExtractor }\end{array}$ & Classification Accuracy \\
& DBNN \\
\hline NDWF10 & $97.76 \%$ & \\
NDWF5 & $96.05 \%$ & $97.70 \%$ \\
NDWF14 & $96.32 \%$ & $98.52 \%$ \\
\hline
\end{tabular}

In such images even visual classification becomes difficult. In order to examine the effects of noise on the classification, we have therefore chosen to degrade the training image NDWF10 by adding progressively higher levels of noise, rather than use additional low $S / N$ data. We have used the IRAF task mknoise to increase the noise level of our training set. The level of noise was controlled by 
Table 6. Number of objects selected in the degraded images. The first line gives values for the undegraded data. The 4 degraded images are in decreasing order of $S / N$ ratio. The criteria for object selection were the same as those for the undegraded training set image NDWF10.

\begin{tabular}{llll}
\hline \hline Image & Background & $\begin{array}{l}\text { Objects with } \\
m_{R}>25\end{array}$ & $\begin{array}{l}\text { Number of objects } \\
\text { Selected }\end{array}$ \\
\hline NDWF10 (undegraded image) & 879.0 & 313 & 402 \\
NDWF104X5 & 175.8 & 313 & 402 \\
NDWF103X5 & 351.6 & 2 & 49 \\
NDWF102X5 & 527.4 & 1 & 37 \\
NDWF10X5 & 703.2 & 0 & 30 \\
\hline
\end{tabular}

Table 7. Classification accuracy of SExtractor and DBNN as the NDWFS image is gradually degraded. Objects that failed with a confidence level greater than $60 \%$ are marked as real failures. Number of objects with $R$ magnitude greater than 25 in each set are shown in square brackets.

\begin{tabular}{|l|l|l|l|l|l|l|l|l|}
\hline Image & \multicolumn{2}{|l|}{ Marginal Objects } & \multicolumn{2}{l|}{ Marginally Passed } & \multicolumn{2}{l|}{ Marginally Failed } & \multicolumn{2}{c|}{ Real failures } \\
\hline & DBNN & SEx & DBNN & SEx & DBNN & SEx & DBNN & SEx \\
\hline NDWF10 & 31 & 34 & $21[17]$ & $31[28]$ & $10[8]$ & $3[3]$ & 0 & $6[3]$ \\
NDWF104X5 & 31 & 34 & $21[17]$ & $31[28]$ & $10[8]$ & $3[3]$ & 0 & $6[3]$ \\
NDWF103X5 & 4 & 3 & $3[0]$ & $3[0]$ & $1[0]$ & 0 & 0 & $1[0]$ \\
NDWF102X5 & 5 & 2 & $2[0]$ & $2[0]$ & $3[0]$ & 0 & 0 & $1[0]$ \\
NDWF10X5 & 1 & 1 & 0 & 1 & $1[0]$ & 0 & $1[0]$ & 0 \\
\hline
\end{tabular}

using progressively higher values for background counts. The original image has a background of 879 counts. Four additional images were created having a background count of $20 \%, 40 \%, 60 \%$ and $80 \%$ of the original background. mknoise was used to add Poisson noise to each of these 4 images, and they represent progressively higher levels of background noise and lower $S / N$ ratio as compared to the original image. Note that the noise being added by us here is in addition to the noise introduced during the acquisition of the NDWFS data (which is already present in the original undegraded image). Sources are extracted from the degraded images with the same SExtractor parameters used for the original training set. The number of sources found in the degraded images are listed in 6 . As expected, the noisier the image, the lower was the number of objects selected. The DBNN was not retrained; sources in the degraded images were classified using the DBNN trained with the original training set.

We have listed in Table 7 the performance of SExtractor and DBNN on the degraded images. We find that DBNN performance is slighter poorer than that of SExtractor on the fainter sources. This may be due to the fact that SExtractor uses the magnitudes at 8 different isophotes as input parameters while DBNN looks for gradients. For fainter objects, gradients are smaller, making DBNN fail for a few faint objects. A factor in favour of DBNN is that it was trained with possibly contaminated training data (due to limitations of the humans who constructed the training and test sets) and can be retrained, while for SEx, the training data was pristine (simulated) and frequent retraining is not practical.
The second observation from the table is that, at brighter magnitudes, DBNN produces more accurate classification on marginal objects compared to SEx. Also on objects that produce high confidence levels, results from DBNN are marginally better than those of SExtractor. It is important to keep in mind that the the confidence levels reported by a neural network do not indicate the difficulty in visual classification by humans. The confidence levels are parameter dependent and merely quantify the appropriateness of a set of parameters. The actual measures of efficiency of a classifier are (1) the total number of objects it can classify with good confidence; a good classifier should have a minimum number of marginal objects at all magnitudes and (2) it should produce minimum errors at high confidence levels. The table shows that DBNN does at least as well as SExtractor in overall efficiency of classification on both these counts.

\section{Discussion and summary}

The results from the test data sets above indicate that the DBNN classifier works as well as the widely used SExtractor software. All instances of DBNN failure correspond to objects that do not have a counterpart in the training set or objects that are difficult (but not impossible) to classify visually. In both test sets, there is no instance where an obvious misclassification has occurred.

As mentioned before, in addition to the consistency, the increase in speed of the training process is very significant here. Our training procedure for DBNN on the 402 objects in the training dataset took $0.23 \mathrm{~s}$ on an 
Intel Pentium III processor running at a clock speed of $700 \mathrm{MHz}$. Such short training times are invaluable when one has to optimally deal with large datasets that are collected and processed over a significantly wide span of time, demanding repeated retraining of the classifier to account for variations in observing conditions and use of newer and better parameters for classification. Data from large surveys fall into this category. Also, unlike the back propagation neural network (BPNN), since DBNN is based on Bayesian probability estimates, it is immune to diverse training vectors that often appear in the training set due to noise in the observation. This means that the performance of the network is likely to be significantly better than the BPNN beyond the completeness limit.

In this paper we have illustrated the power of the technique by applying it to the star galaxy classification problem. The technique can easily be applied to all classification problems that currently employ BPNN. For example, by using the large number of photometric and spectroscopic parameters measured (for millions of objects) by surveys such as the the Sloan Digital Sky Survey, it will be possible to apply the DBNN technique to identify interesting samples for study in the vast, largely unexplored parameter space. We are in the process of enhancing DBNN to solve problems that involve regression.

The source code, documentation and the training and test set images described in this paper may be downloaded from the URL: http://www.iucaa.ernet.in/ nspp/ dbnn.html

Acknowledgements. The authors would like to thank Ashish Mahabal for discussions and a careful reading of the manuscript. The first author would like to express his sincere thanks to Inter University Center for Astronomy and Astrophysics and the computer staff there for providing him all the required facilities for the successful completion of this project. We also thank the anonymous referee whose detailed comments considerably improved this paper.

This work made use of images and data products provided by the NOAO Deep Wide-Field Survey (Jannuzi \& Dey 1999), which is supported by the National Optical Astronomy
Observatory (NOAO). NOAO is operated by AURA, Inc., under a cooperative agreement with the National Science Foundation.

\section{References}

Andreon, S., Gargiulo, G., Longo, G., Tagliaferri, R., \& Capuano, N. 2000, MNRAS, 319, 700

Bayes, T. 1763, Philosophical Transactions of the Royal Society of London, 53, 370

Bertin, E., \& Arnouts, S. 1996, A\&AS, 117, 393

Bishop, C. M. 1999, Neural Networks for Pattern Recognition (Oxford: Oxford University Press)

Elkan, C. 1997, Boosting and Naive Bayesian Learning, Technical Report No.CS97-557 (San Diego: University of California)

Folkes, S. R., Lahav, O., \& Maddox, S. J. 1996, MNRAS, 283, 651

Jannuzi, B. T., \& Dey, A. 1999, in Photometric Redshifts and the Detection of High Redshift Galaxies, ed. R. Weymann et al., ASP Conf. Ser., 191, 111

Kohonen, T. 1982, Biological Cybernetics, 44, 135

Kohonen, T. 1995, Self-Organizing Maps (Berlin:Springer)

Laplace, P. S. 1812, Theorie Analytique des Probabilities (Paris: Courcier)

Loredo, T. J. 1990, in Maximum Entropy and Bayesian Methods, ed. P. F. Fougere (Dordrecht: Kluwer), 81

McClelland, J. L., \& Rumelhart, D. E. 1989, Explorations in Parallel Distributed Processing (Cambridge: MIT Press)

Miller, A. S., \& Coe, M. J. 1996, MNRAS, 279, 293

Odewahn, S. C., Stockwell, E. B., Pennington, R. L., Mumphreys, R. M., \& Zumach, W. A. 1992, AJ, 103, 1

Philip, N. S., \& Joseph, K. B. 2001, Journal of Intelligent Data Analysis, 4, 6

Rogers, R. D., \& Riess, A. G. 1994, PASP, 106, 532

Schiffmann, W., Joost, M., \& Werner, R. 1994, Optimization of the Back Propagation Algorithm for Multilayer Perceptrons in The Neuroprose Archive

Singh, H. P., Gulati, R. K., \& Gupta, R. 1998, MNRAS, 295, 312

Weir, N., Fayyad, U. M., \& Djorgovski, S. 1995, AJ, 109, 2401 